\title{
Stability of an autologous platelet clot in the pericardial sac: An experimental and clinical study
}

\author{
Leonard M. Rademakers, MD, ${ }^{\mathrm{a}}$ Paul F. Gründeman, MD, PhD, ${ }^{\mathrm{b}}$ Robert W. Bolderman, MD, ${ }^{\mathrm{a}}$ Frederik H. van der Veen, PhD, ${ }^{\mathrm{a}}$ \\ and Jos G. Maessen, MD, $\mathrm{PhD}^{\mathrm{a}}$
}

\begin{abstract}
Objective: Autologous platelet clots serve as slow-release delivery systems for platelet-derived growth factors and cytokines. Their application to the pericardial sac might facilitate salvage and repair of ischemically injured myocardium. However, little is known about platelet clot stability in the pericardial sac. We investigated the stability of platelet clots in vitro and after administration to the pericardial sac in pigs and patients.
\end{abstract}

\begin{abstract}
Methods: In 5 Yorkshire-Landrace pigs and 10 patients, in vitro manufactured autologous platelet gel (Medtronic Magellan Platelet Separator) and platelet-rich fibrin (Vivolution Vivostat System) were administered to the pericardial sac for 30 minutes. Two antifibrinolytics (tranexamic acid and aprotinin) were tested for their capacity to stabilize autologous platelet gel. In vitro clots, incubated at $37^{\circ} \mathrm{C}$ for 48 hours, served as controls. Clot weight was measured before and after administration.
\end{abstract}

Results: In vitro, autologous platelet gel clots of either formula liquefied almost entirely within 60 minutes whereas platelet-rich fibrin clots remained intact. In the pig, platelet clot weight decreased to $16.7 \% \pm 7.8 \%$ $(P<.05)$ and $66.4 \% \pm 3.2 \%(P<.05)$ of initial clot weight for autologous platelet gel and platelet-rich fibrin, respectively. Addition of antifibrinolytics to autologous platelet gel did not reduce clot degradation significantly. In patients, autologous platelet gel and platelet-rich fibrin clot weight remained $9.0 \% \pm 1.5 \%(P<.05)$ and $73.7 \% \pm 2.6 \%(P<.05)$ of initial clot weight, respectively.

Conclusions: Autologous platelet gel is unstable both in vitro and in vivo, whereas platelet-rich fibrin remains intact in vitro and, compared with autologous platelet gel, is less subject to degradation in pigs and in patients.

The pericardium serves as a biochemical active microenvironment and interacts with the coronaries and the myocardium. ${ }^{1,2}$ Therefore, the pericardial space is considered to be a potential ideal site for local drug therapy for myocardium and coronaries. Intrapericardial drug delivery has advantages over systemic administration: it provides higher intramural concentrations, has fewer side effects that may accompany systemic administration, is highly reproducible, and has a high efficiency of localization. ${ }^{3,4}$ Furthermore, drug delivery into a low-turnover reservoir may prevent a rapid washout of agents.

Platelets contain storage pools of various growth factors and cytokines. ${ }^{5}$ An increased understanding of the role of platelets in wound healing led to the idea of using platelets as "therapeutic vehicles." In various tissues, platelets trapped in a fibrin clot proved to serve as a site-specific slowrelease delivery system. ${ }^{6-8}$ Hypothetically, application of platelet clots to ischemically injured myocardium might

From the Cardiovascular Research Institute Maastricht (CARIM), Department of Cardiothoracic Surgery, Maastricht University Medical Center, ${ }^{a}$ Maastricht, The Netherlands, and Laboratory of Experimental Cardiology, Division of Heart and Lungs, University Medical Center Utrecht, ${ }^{\mathrm{b}}$ Utrecht, The Netherlands.

Received for publication July 18,2008 ; revisions received Sept 15, 2008; accepted for publication Oct 9, 2008.

Address for reprints: Leonard M. Rademakers, MD, Maastricht University Medical Center, Department of Cardiothoracic Surgery, P. Debyelaan 25, 6229 HX Maastricht, The Netherlands (E-mail: 1.rademakers@ctc.unimaas.nl).

J Thorac Cardiovasc Surg 2009;137:1190-4

$0022-5223 / \$ 36.00$

Copyright (c) 2009 by The American Association for Thoracic Surgery doi:10.1016/j.jtcvs.2008.10.012 enhance salvage and repair. ${ }^{9}$ However, little is known about the stability of platelet clots when administered to the pericardial sac. This study assessed the stability of 4 different platelet clot compositions over time in pigs and in patients. In vitro clots served as controls.

\section{MATERIALS AND METHODS \\ Preparation and Application of Autologous Platelet Gel and Platelet-Rich Fibrin Clots}

Animal experiments were performed in accordance with the Guide for the Care and Use of Laboratory Animals, as approved by the National Research Council (National Institutes of Health), and were approved by the committee on the care and use of experimental animals at our institute. Similarly, clinical experiments were approved by the medical ethical committee of our institution, and all patients provided informed consent.

Venous blood was freshly obtained from pigs and patients. To monitor clot dynamics properly, autologous platelet gel (APG) and platelet-rich fibrin (PRF) clots were manufactured in vitro in a standardized way: platelet clots with $2.7-\mathrm{cm}$ diameter and approximately $1-\mathrm{cm}$ height were manufactured in plastic sterile sample cups. Before application to the pericardial sac, initial clot weight was measured (Figure 1).

The Magellan Autologous Platelet Separator System and Disposables Kit (Medtronic Inc, Minneapolis, MN) were used to manufacture plateletrich plasma (PRP) and thrombin. A detailed description of the PRP preparation method is available at the manufacturer and can be found elsewhere. ${ }^{10}$ In short, $52 \mathrm{~mL}$ of whole blood was drawn into a $60-\mathrm{mL}$ syringe, preaspired with $8 \mathrm{~mL}$ of anticoagulant citrate dextrose $(0.022 \mathrm{~g} / \mathrm{mL}$ sodium citrate). The syringe was processed to yield $8 \mathrm{~mL}$ of PRP, $3 \mathrm{~mL}$ of which was used to generate $2.5 \mathrm{~mL}$ of thrombin. Thrombin activator reagents include glass fiber and calcium chloride $10 \%$. The final thrombin activity approximated 10 to $15 \mathrm{IU} / \mathrm{mL} .{ }^{11}$ The factor of increase in platelet concentration over baseline whole blood level depends on the chosen volume PRP to be produced. According to the manufacturer, in our study the platelet 


\section{Abbreviations and Acronyms \\ $\mathrm{APG}=$ autologous platelet gel \\ $\mathrm{BU}=$ batroxobin units \\ $\mathrm{PRF}=$ platelet-rich fibrin \\ PRP $=$ platelet-rich plasma}

concentration would approximate 6 times the baseline whole blood level (minimum platelet count $1000 \times 10^{3} / \mathrm{mm}^{3}$ ). Regardless of chosen PRP amount, PRP fibrin(ogen) content remains at whole blood level (averaging $2.3 \mathrm{mg} / \mathrm{mL}$ ). Subsequently, the platelet concentrate was mixed with thrombin. This induced activation of platelets and resulted in the formation of an APG. Two fibrinolysis inhibitors, aprotinin and tranexamic acid, were tested for their capacity to stabilize APG. Aprotinin acts as a reversible, competitive inhibitor of plasmin. Tranexamic acid inhibits plasminogen activation by preventing binding of plasminogen to fibrin. Before platelet activation, either $30 \mathrm{mg} / \mathrm{mL}$ tranexamic acid (Cyklokapron; Pharmacia and Upjohn $\mathrm{AB}$, Stockholm, Sweden) or $3000 \mathrm{kIU} / \mathrm{mL}$ aprotinin (Trasylol; Bayer, Leverkusen, Germany) were mixed with PRP.

The Vivostat Processor Unit (Vivolution A/S, Birkeroed, Denmark) and the Vivostat PRF Preparation Kit were used to prepare PRF. PRF is an autologously derived tissue sealant in which platelets get trapped. A detailed description of the production process is available at Vivolution $\mathrm{A} / \mathrm{S}$ and can be found elsewhere. ${ }^{12}$ In short, $120 \mathrm{~mL}$ of whole blood was drawn into the collecting unit, which was preaspired with acid-citrate solution containing tranexamic acid $(100 \mathrm{mg}$ ) and centrifuged. Subsequently, the PRP was converted into an acid-soluble fibrin I polymer by the addition of batroxobin (30 batroxobin units $[\mathrm{BU}]$ in the device, of which 6 to $9 \mathrm{BU}$ remain in the PRF after processing). The snake venom Batroxobin catalyzes the release of fibrinopeptide A from fibrinogen without activating coagulation factor XIII. The fibrin I polymer and the platelets were isolated by centrifugation and dissolved in $0.2 \mathrm{~mol} / \mathrm{L}$ acetate buffer $(\mathrm{pH} 4)$. About $6 \mathrm{~mL}$ of Vivostat PRF comprising cross-linked fibrin II polymer enriched with the platelets was obtained through neutralization of 7 parts of the acidic fibrin I solution with 1 part of a $0.75 \mathrm{~mol} / \mathrm{L}$ carbonate/bicarbonate buffer ( $\mathrm{pH} 10)$. Polymerization is mediated by activation of endogenous prothrombin to thrombin that activates endogenous factor XIII. According to the manufacturer, the platelet concentration would approximate 10 times to baseline whole blood level (minimum platelet count $1200 \times 10^{3} / \mathrm{mm}^{3}$ ), whereas fibrin concentration averages $14 \mathrm{mg} / \mathrm{mL}$.

\section{In Vitro Model}

Parallel to each porcine experiment, in vitro clots (ie, APG, APG plus tranexamic acid, APG plus aprotinin, and PRF) were manufactured. All clots were placed in closed, plastic test tubes and incubated in a stove at $37^{\circ} \mathrm{C}$ for 48 hours.

\section{Animal Model}

Five Yorkshire-Landrace pigs (weight ranged from 60 to $75 \mathrm{~kg}$ ) were sedated (ketamine $10 \mathrm{mg} / \mathrm{kg}$ intramuscularly, midazolam $0.5 \mathrm{mg} / \mathrm{kg}$ intramuscularly, and atropine $0.04 \mathrm{mg} / \mathrm{kg}$ intramuscularly), anesthetized (thiopental sodium, $5 \mathrm{mg} / \mathrm{kg}$ intravenously), intubated, and mechanically ventilated (mixture of oxygen and air 1:1). Anesthesia was maintained with midazolam $\left(1.0 \mathrm{mg} \cdot \mathrm{kg}^{-1} \cdot \mathrm{h}^{-1}\right.$ intravenously), sufentanyl citrate $\left(4 \mu \mathrm{g} \cdot \mathrm{kg}^{-1} \cdot \mathrm{h}^{-1}\right.$ intravenously), and pancuronium bromide $\left(0.1 \mathrm{mg} \cdot \mathrm{kg}^{-1} \cdot \mathrm{h}^{-1}\right.$ intravenously). Euthanasia was obtained with pentobarbital sodium $(20 \mathrm{mg} / \mathrm{kg}$ intravenously). Electrocardiography was used to monitor heart rate, rhythm, and ST-segment changes during the surgical procedures. Peak and mean arterial and central venous pressures, end-expiratory $\mathrm{CO}_{2}$, pulse oxymetry, blood gases (ie, $\mathrm{pH}, \mathrm{pCO}_{2}$ ) were monitored during surgery. The pigs were kept warm by an external heating mattress to maintain a rectal temperature of

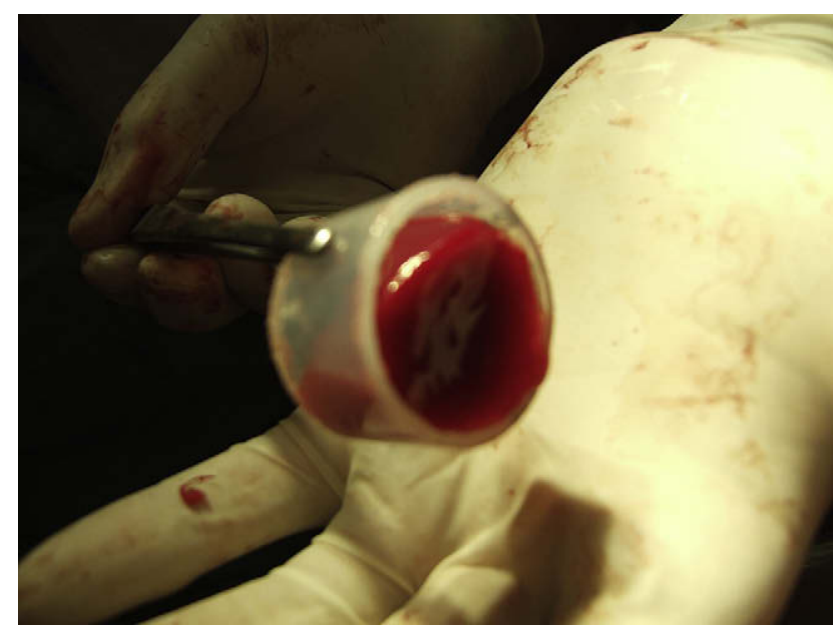

FIGURE 1. In vivo incubation of autologous platelet clot. Freshly manufactured autologous platelet gel clot in plastic test container just before administration to the pericardial sac.

$38^{\circ} \mathrm{C}$ to $39^{\circ} \mathrm{C}$. Sodium-chloride solution, 0.2 to $0.35 \mathrm{~mL} \cdot \mathrm{kg}^{-1} \cdot \mathrm{min}^{-1}$, was given intravenously. All pigs underwent a median sternotomy. After making a 4- to 5-cm-long incision in the pericardium, freshly manufactured clots were left behind in the lowest part of the pericardial sac. The pericardial incision was approximated with suture wires during incubation. The clot residue was taken out after 30 minutes and clot weight was measured. Each animal consecutively received APG, APG plus tranexamic acid, APG plus aprotinin, and PRF. After removal of the clot residue, clot weight was measured. All pericardial fluid was removed and the pericardial sac was rinsed with saline. Redundant saline was removed and a washout period of 1 hour was applied before administering a new platelet clot.

\section{Patient Model}

To test whether results obtained from animal studies can be extrapolated to humans, 10 patients ( 6 men; median age 74 , range 61 to 81 ) undergoing elective left internal thoracic artery-to-left anterior descending coronary artery bypass grafting, were included in this study. Five patients received an APG clot and 5 patients, a PRF clot. Exclusion criteria included antiplatelet therapy (unless stopped 10 days previous to surgery), anemia (hemoglobin $<13 \mathrm{~g} / \mathrm{dL}$ in men and $<12 \mathrm{~g} / \mathrm{dL}$ in women), platelet count below $150 \times 10^{3} /$ $\mathrm{mm}^{3}$, and fibrinolytic therapy (unless stopped 2 days previous to surgery). In the operating room, venous blood was withdrawn and platelet clots were manufactured. While harvesting the left internal thoracic artery, the platelet clot was administered to the lowest part of the pericardial sac through a 4- to 5 -cm-long incision. The pericardial incision was approximated with suture wires during incubation. After 30 minutes, the clot was taken out. Before and after incubation, clot weight was measured.

\section{Statistical Analyses}

The paired sample Wilcoxon rank-sum test was used to compare differences between initial and residual clot weight as well as differences between various APG compositions and PRF. Unless stated otherwise, data are expressed as mean \pm standard deviation. Statistical analyses were carried out using SPSS software, version 12.0 (SPSS Inc, Chicago, IL).

\section{RESULTS}

In vitro, APG, APG plus tranexamic acid, and APG plus aprotinin liquefied almost completely within 60 minutes of incubation, whereas PRF clots remained intact for (at least) 48 hours (Figure 2). 


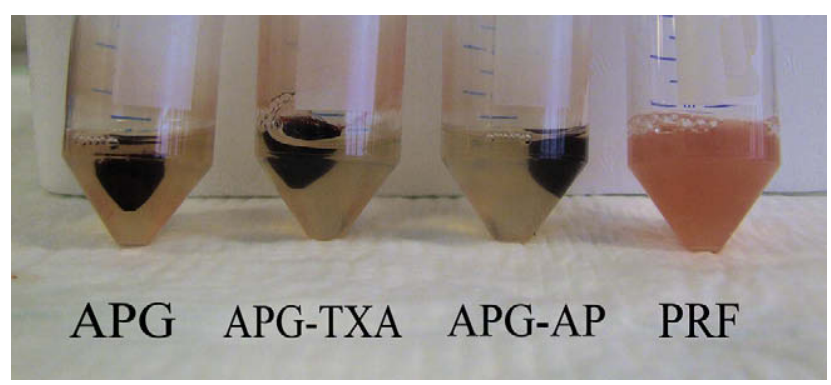

FIGURE 2. In vitro incubation of autologous platelet clots. After $60 \mathrm{~min}$ utes of incubation at $37^{\circ} \mathrm{C}$, the PRF clot remained solid while either APG clot formula liquefied almost completely. $A P G$, Autologous platelet gel; $A P G-T X A$, APG plus tranexamic acid; $A P G-A P$, APG plus aprotinin; $P R F$, platelet-rich fibrin.

Administration of platelet clots to the pericardial sac did not result in changes in heart rate and rhythm or ST-segments. In addition, no changes in systolic and diastolic blood pressure were recorded upon clot administration.

In pigs, after 30 minutes of intrapericardial incubation, $16.7 \% \pm 7.8 \%(P<.05)$ of initial APG clot weight remained (Table 1). Addition of tranexamic acid or aprotinin to APG resulted in $19.5 \% \pm 9.8 \%$ and $15.6 \% \pm 8.6 \%$ clot weight residue, respectively (both $P<.05$ ). Compared with either APG formula, less clot weight reduction occurred when PRF was administered to the pericardial sac (ie, $66.4 \% \pm 3.2 \%$ of initial clot weight remained; $P<$ $.05)$. In 1 porcine PRF experiment, the incubation period was extended to 4 hours. After the first 30 minutes, clot weight was measured hourly. After 30 minutes, clot weight was reduced to $61 \%$ of initial clot weight, and during the consecutive 3.5 hours, clot weight was further reduced to approximately $42 \%$ of initial clot weight.

In patients, after 30 minutes of incubation, $9.0 \% \pm 1.5 \%$ and $73.7 \% \pm 2.6 \%$ (both $P<.05$ ) of initial APG and PRF clot weight remained, respectively (Table 1).

\section{DISCUSSION}

The main findings of this study are: (1) in vitro, APG clots of either composition liquefy almost completely within 60 minutes, although PRF clots remain intact for at least 48 hours; (2) application of platelet clots to the pericardial sac does not impair cardiac function; (3) in pigs and in patients, and irrespective of clot composition, APG is unstable; (4)
PRF degrades significantly less compared with APG, both in pigs and in patients.

In vitro experiments were designed to investigate exclusively physical clot stability. Other potential causes of degradation that take place in vivo, such as mechanical stress and fibrinolysis, were thus precluded. All APG clots almost completely liquefied within a relatively short period of time. Clot retraction (ie, the physiologic process of serum expression out of a fibrin matrix due to the contractile properties of activated platelets) may be a plausible explanation of this observation. However, PRF did not show signs of clot retraction within 48 hours of incubation. Batroxobin-induced fibrin polymerization may account for this difference. In contrast to thrombin (which is used to induce gelation in APG), batroxobin does not activate platelets that are trapped in the fibrin network. ${ }^{13,14}$ Because clot retraction is a process exhibited by activated platelets, a marked delay in clot retraction may be expected upon batroxobin induced gelation. ${ }^{15}$

In pigs and patients, no adverse reactions upon application of platelet clots to the pericardial sac were observed.

In both animals and patients, APG clot weight decreased significantly to less than $20 \%$ and $10 \%$ of initial clot weight after 30 minutes of incubation, respectively. Although the exact cause of APG clot degradation in vivo is not completely understood, there may be some other potential contributors to platelet clot degradation besides the aforementioned clot retraction. Because the mechanical strength of fibrin clots is directly related to their fibrin concentration, low fibrin levels in APG clots may have contributed to rapid degradation. Buchta and colleagues ${ }^{16}$ and Christensen and associates ${ }^{10}$ performed in vitro analyses on the platelet-free Vivostat formula and Magellan PRP, and they measured fibrin levels approximating $15 \mathrm{mg} / \mathrm{mL}$ and $3 \mathrm{mg} / \mathrm{mL}$, respectively. Indeed, in our experience, PRF clots felt less "jelly" when compared with APG clots. Thus, APG clots may be more vulnerable to mechanical stress in the pericardial sac, relative to PRF clots. In addition, mesothelial cells lining the pericardium are known for their capacity to secrete fibrinolytic factors into the pericardial sac. ${ }^{1,17,18}$ Thus, a high fibrinolytic activity may have contributed to fibrin clot degradation as well. Coadministration of antifibrinolytic drugs to APG did not reduce the amount of fibrin clot degradation. Probably, clot retraction as well as fibrin matrix weakness may have prevented antifibrinolytics from showing their real potential in slowing down fibrinolysis.

TABLE 1. Pericardial platelet clot weight in pigs and patients

\begin{tabular}{|c|c|c|c|c|c|c|}
\hline & $\begin{array}{c}\text { APG } \\
(\operatorname{pig}, \mathbf{n}=\mathbf{5})\end{array}$ & $\begin{array}{c}\text { APG } \\
(\text { patient, } \mathbf{n}=\mathbf{5})\end{array}$ & $\begin{array}{c}\text { APG }+ \text { tranexamic acid } \\
(\text { pig, } \mathbf{n}=\mathbf{5})\end{array}$ & $\begin{array}{c}\text { APG }+ \text { aprotinin } \\
(\mathbf{p i g}, \mathbf{n}=\mathbf{5})\end{array}$ & $\begin{array}{c}\text { PRF } \\
(\text { pig, }, \mathbf{n}=5) \\
\end{array}$ & $\begin{array}{c}\text { PRF } \\
(\text { patient, } \mathbf{n}=5)\end{array}$ \\
\hline Initial clot weight (g) & $4.6 \pm 0.9$ & $6.7 \pm 0.3$ & $5.6 \pm 0.3$ & $5.6 \pm 0.3$ & $6.4 \pm 0.4$ & $6.6 \pm 0.2$ \\
\hline Residue clot weight (g) & $0.8 \pm 0.5^{*}$ & $0.6 \pm 0.1^{*}$ & $1.1 \pm 0.5^{*}$ & $0.9 \pm 0.4^{*}$ & $4.3 \pm 0.4 * \dagger$ & $4.8 \pm 0.2 * \ddagger$ \\
\hline Residue clot weight $(\%)$ & $16.7 \pm 7.8$ & $9.0 \pm 1.5$ & $19.5 \pm 9.8$ & $15.6 \pm 8.6$ & $66.4 \pm 3.2$ & $73.7 \pm 0.3$ \\
\hline
\end{tabular}

$A P G$, Autologous platelet gel; $P R F$, platelet-rich fibrin. *Compared with initial clot weight $(P<.05)$. †Compared with either porcine APG clot formula $(P<.05)$. $\ddagger$ Compared with residue clot weight of clinical APG group $(P<.05)$. 
Both in pigs and in patients, PRF clots degraded to a more or less similar extent. However, clot weight degradation was significantly less than either APG clots. As discussed above, due to their different manufacturing process, PRF clots are less subject to clot retraction. In addition, PRF clots contain a higher fibrin level and may therefore better cope with the mechanical stress in the pericardial sac. However, in 1 extended observation time of 4 hours, PRF clots degraded to about $42 \%$ of initial clot weight. Probably, a combination of high pericardial fibrinolytic activity and intrapericardial mechanical stress may have accounted for extensive platelet clot degradation over time.

In addition to previously mentioned factors potentially influencing platelet clot stability, there may be several others. Differences in concentration of (non)activated coagulation factors, platelet count and platelet (dys)function, for example, due to high fibrin split product levels, may also affect platelet clot stability.

Although the life span of APG and PRF clots in the pericardial sac is rather limited, these and other fibrin-based matrices proved to be effective site-specific slow-release delivery systems for drugs, ${ }^{19}$ growth factors, ${ }^{20}$ or stem cells ${ }^{21}$ within various surgical specialties (eg, oral-maxillofacial, orthopedic, and general surgery) ${ }^{22}$ and within the treatment of chronic skin and soft tissue ulcers. ${ }^{23}$ Slow-release delivery of growth factors in the pericardial sac may enhance neovascularization of ischemically damaged myocardium. Similarly, stem cells may be used to target cardiac repair. Platelet clots loaded with antiarrhythmic agents, for example, can be used to treat postoperative atrial fibrillation.

As discussed earlier, there may be several potential causes of rapid fibrin matrix degradation. Quick degradation of fibrin clots may implicate that coadministered therapeutics will be released within a short period of time. For shortterm purposes, PRF may be a suitable carrier for drug delivery; however, a gradual and long-term release of therapeutics in the pericardial sac may require a scaffold that better resists these attacks. With recent developments in biotechnology and bioengineering, several alternative biodegradable drug delivery systems are becoming available. ${ }^{24,25}$

This study has several limitations. The intention of slowrelease drug delivery systems is to gradually release therapeutics over a certain time span. However, platelet clots were exposed to the pericardial sac for a very short period of time, which may probably not reflect their intended use in clinical practice. Nevertheless, despite a limited incubation time in vivo, we were able to assess whether tested platelet clots may be a suitable slow-release drug delivery system for intrapericardial application.

We assumed a correlation between platelet clot weight reduction and the release of therapeutics. In some situations, this may not hold true. If therapeutics are not trapped in or bound to the fibrin network, they might diffuse out the clot. Also, upon clot retraction, theoretically only serum might be expressed out of the fibrin matrix, leaving the therapeutics inside. In this case, actual clot weight may decrease when therapeutics are not released. To better study causes of platelet clot degradation or drug release, labeling of fibrinogen in the platelet clot as well as labeling of the trapped therapeutic might be an option. The former may enable assessment of the exact causes of platelet clot degradation, and the latter may help reveal whether intrapericardial platelet clot weight reduction correlates to release of tested therapeutic.

Furthermore, the (fixed) sequence of applying different platelet clots to the pericardial sac may have influenced results. Residual thrombin or batroxobin, or intrinsic activators, may have influenced the antifibrinolytic properties of tested agents. By rinsing the pericardial sac thoroughly with saline and applying a washout period of 1 hour, we empirically aimed to minimize carryover effects. However, whether a 1-hour washout period is sufficient to avoid carryover effects remains to be investigated.

Safety of intrapericardial platelet clot application should be assessed more thoroughly before it can be clinically adopted. Although platelet clots are relatively small and mould easily in the pericardial sac, they might compress (and thereby injure) epicardial tissue or affect graft patency. The latter might also be affected due to potential hyperproliferation at the anastomotic sites due to release of therapeutics. Based on monitoring of heart rate and rhythm and blood pressure, we assessed hemodynamic safety of intrapericardial platelet clot application. However, more sophisticated techniques, such as echocardiography, may be used to further evaluate effects of platelet clots on regional wall motion.

In summary, this study investigated the stability of 4 different autologous platelet clot compositions when administered to the pericardial sac, and in vitro clots served as controls. APG in either composition is unstable both in vitro and in vivo, whereas PRF remains intact in vitro for a longer period of time and is less subject to degradation in animals and patients.

We gratefully acknowledge Monique de Jong and Theo van der Nagel for their technical assistance during experiments. We are indebted to Vivolution (Vivolution A/S, Birkeroed, Denmark) and Alfons Balvers (Cardiac Care, Herkenbosch, The Netherlands) for providing Vivostat equipment. Furthermore, we wish to thank Medtronic (Medtronic Bakken Research Center, Maastricht, The Netherlands) for providing the Magellan System and disposables.

\section{References}

1. Spodick DH. Physiology of the normal pericardium: functions of the pericardium. In: Spodick DH, ed. The pericardium: A comprehensive textbook. New York Marcel Dekker; 1997. p. 15-26.

2. Laham RJ, Hung D, Simons M. Therapeutic myocardial angiogenesis using percutaneous intrapericardial drug delivery. Clin Cardiol. 1999;22(1 Suppl. 1):I6-9.

3. Laham RJ, Rezaee M, Post M, Xu X, Sellke FW. Intrapericardial administration of basic fibroblast growth factor: myocardial and tissue distribution and comparison with intracoronary and intravenous administration. Catheter Cardiovasc Interv. 2003;58:375-81. 
4. Stoll HP, Carlson K, Keefer LK, Hrabie JA, March KL. Pharmacokinetics and consistency of pericardial delivery directed to coronary arteries: direct comparison with endoluminal delivery. Clin Cardiol. 1999;22(1 Suppl. 1):I10-6.

5. Pietrzak WS, Eppley BL. Platelet rich plasma: biology and new technology. J Craniofac Surg. 2005;16:1043-54.

6. Currie LJ, Sharpe JR, Martin R. The use of fibrin glue in skin grafts and tissueengineered skin replacements: a review. Plast Reconstr Surg. 2001;108:1713-26.

7. Tabata S, Eckert HG, Kellner HM, Hayashi S, Fujimoto K. Pharmacokinetic study of a fibrin adhesive agent, Beriplast in rats. Oyo Yakuri-Pharmacometrics. 1986;31:1123-7.

8. Yazawa M, Ogata H, Nakajima T, Mori T, Watanabe N, Handa M. Basic studies on the clinical applications of platelet-rich plasma. Cell Transplant. 2003;12: 509-18.

9. Mogan C, Larson DF. Rationale of platelet gel to augment adaptive remodeling of the injured heart. J Extra Corpor Technol. 2004;36:191-6.

10. Christensen K, Vang S, Brady C, Isler J, Allen K, Anderson J, Holt D. Autologous platelet gel: an in vitro analysis of platelet-rich plasma using multiple cycles. $J E x$ tra Corpor Technol. 2006;38:249-53.

11. Everts PA, Knape JT, Weibrich G, Schonberger JP, Hoffmann J, Overdevest EP, Box HA, van Zundert A. Platelet-rich plasma and platelet gel: a review. J Extra Corpor Technol. 2006;38:174-87.

12. Lundquist R, Dziegiel MH, Agren MS. Bioactivity and stability of endogenous fibrogenic factors in platelet-rich fibrin. Wound Repair Regen. 2008;16:356-63.

13. Niewiarowski S, Stewart GJ, Nath N, Sha AT, Lieberman GE. ADP, thrombin, and Bothrops atrox thrombinlike enzyme in platelet-dependent fibrin retraction. Am J Physiol. 1975;229:737-45.

14. Hantgan RR, Taylor RG, Lewis JC. Platelets interact with fibrin only after activation. Blood. 1985;65:1299-311.

15. Mazzucco L, Balbo V, Cattana E, Borzini P. Platelet-rich plasma and platelet gel preparation using Plateltex(R). Vox Sang. 2008;94:202-8.
16. Buchta C, Hedrich HC, Macher M, Hocker P, Redl H. Biochemical characterization of autologous fibrin sealants produced by CryoSeal and Vivostat in comparison to the homologous fibrin sealant product Tissucol/Tisseel. Biomaterials. 2005;26:6233-41.

17. Porter JM, Ball AP, Silver D. Mesothelial fibrinolysis. J Thorac Cardiovasc Surg. 1971;62:725-30.

18. Porter JM, Ball AP, Silver D. The mechanism of blood clot resorption from mesothelial spaces. Surg Forum. 1971;22:83-4.

19. Lardinois D, Jung FJ, Opitz I, Rentsch K, Latkoczy C, Vuong V, et al. Intrapleural topical application of cisplatin with the surgical carrier Vivostat increases the local drug concentration in an immune-competent rat model with malignant pleuromesothelioma. J Thorac Cardiovasc Surg. 2006;131:697-703.

20. Ishii I, Mizuta H, Sei A, Hirose J, Kudo S, Hiraki Y. Healing of full-thickness defects of the articular cartilage in rabbits using fibroblast growth factor- 2 and a fibrin sealant. J Bone Joint Surg Br. 2007;89:693-700.

21. Christman KL, Fok HH, Sievers RE, Fang Q, Lee RJ. Fibrin glue alone and skeletal myoblasts in a fibrin scaffold preserve cardiac function after myocardial infarction. Tissue Eng. 2004;10:403-9.

22. Everts PA, Overdevest EP, Jakimowicz JJ, Oosterbos CJ, Schonberger JP, Knape JT, van Zundert A. The use of autologous platelet-leukocyte gels to enhance the healing process in surgery, a review. Surg Endosc. 2007;21:2063-8.

23. Driver VR, Hanft J, Fylling CP, Beriou JM. Autologel Diabetic Foot Ulcer Study Group. A prospective, randomized, controlled trial of autologous platelet-rich plasma gel for the treatment of diabetic foot ulcers. Ostomy Wound Manage. 2006;52:68-74.

24. Nair LS, Laurencin CT. Polymers as biomaterials for tissue engineering and controlled drug delivery. Adv Biochem Eng Biotechnol. 2006;102:47-90.

25. Hou Q, Chau DY, Pratoomsoot C, Tighe PJ, Dua HS, Shakesheff KM, Rose FR. In situ gelling hydrogels incorporating microparticles as drug delivery carriers for regenerative medicine. J Pharm Sci. 2008;97:3972-80. 